\title{
Cardiovascular events after clarithromycin use in lower respiratory tract infections: analysis of two prospective cohort studies
}

\author{
(c) $\frac{(1)(8)}{\mathrm{gy}}$ OC OPEN ACCESS
}

\begin{abstract}
Stuart Schembri consultant respiratory physician ${ }^{1}$, Peter A Williamson consultant respiratory physician $^{2}$, Philip M Short clinical lecturer in respiratory medicine ${ }^{13}$, Aran Singanayagam clinical research fellow ${ }^{4}$, Ahsan Akram clinical research fellow ${ }^{5}$, Joanne Taylor foundation doctor ${ }^{5}$, Anika Singanayagam clinical research fellow ${ }^{6}$, Adam T Hill consultant respiratory physician ${ }^{5}$ honorary senior lecturer ${ }^{7}$, James $D$ Chalmers clinical lecturer in respiratory medicine ${ }^{13}$
\end{abstract}

${ }^{1}$ Ninewells Hospital, Dundee DD1 9SY, UK; ${ }^{2}$ Perth Royal Infirmary, Perth PH1 1NX, UK; ${ }^{3}$ University of Dundee, Dundee, UK; ${ }^{4}$ Imperial College London, London SW7 2AZ, UK; ${ }^{5}$ Royal Infirmary of Edinburgh, Edinburgh EH16 4SA, UK; ${ }^{6}$ Chase Farm and Barnet Hospitals NHS Trust, London E1 1BB, UK; ${ }^{7}$ University of Edinburgh, Edinburgh, UK

\begin{abstract}
Objective To study the association of clarithromycin with cardiovascular events in the setting of acute exacerbations of chronic obstructive pulmonary disease and community acquired pneumonia.

Design Analysis of two prospectively collected datasets.

Setting Chronic obstructive pulmonary disease dataset including patients admitted to one of 12 hospitals around the United Kingdom between 2009 and 2011; Edinburgh pneumonia study cohort including patients admitted to NHS Lothian Hospitals between 2005 and 2009.

Population 1343 patients admitted to hospital with acute exacerbations of chronic obstructive pulmonary disease and 1631 patients admitted with community acquired pneumonia.

Main outcome measures Hazard ratios for cardiovascular events at one year (defined as hospital admissions with acute coronary syndrome, decompensated cardiac failure, serious arrhythmia, or sudden cardiac death) and admissions for acute coronary syndrome (acute ST elevation myocardial infarction, non-ST elevation myocardial infarction, and unstable angina). Secondary outcomes were all cause and cardiovascular mortality at one year.

Results 268 cardiovascular events occurred in the acute exacerbations of chronic obstructive pulmonary disease cohort and 171 in the community acquired pneumonia cohort over one year. After multivariable adjustment, clarithromycin use in acute exacerbations of chronic obstructive pulmonary disease was associated with an increased risk of cardiovascular events and acute coronary syndrome-hazard ratios 1.50 (95\% confidence interval 1.13 to 1.97 ) and 1.67 (1.04 to 2.68). After multivariable adjustment, clarithromycin use in community acquired
\end{abstract}

pneumonia was associated with increased risk of cardiovascular events (hazard ratio $1.68,1.18$ to 2.38 ) but not acute coronary syndrome (1.65, 0.97 to 2.80 ). The association between clarithromycin use and cardiovascular events persisted after matching for the propensity to receive clarithromycin. A significant association was found between clarithromycin use and cardiovascular mortality (adjusted hazard ratio $1.52,1.02$ to 2.26$)$ but not all cause mortality $(1.16,0.90$ to 1.51$)$ in acute exacerbations of chronic obstructive pulmonary disease. No association was found between clarithromycin use in community acquired pneumonia and all cause mortality or cardiovascular mortality. Longer durations of clarithromycin use were associated with more cardiovascular events. Use of $\beta$ lactam antibiotics or doxycycline was not associated with increased cardiovascular events in patients with acute exacerbations of chronic obstructive pulmonary disease, suggesting an effect specific to clarithromycin.

Conclusions The use of clarithromycin in the setting of acute exacerbations of chronic obstructive pulmonary disease or community acquired pneumonia may be associated with increased cardiovascular events. These findings require confirmation in other datasets.

\section{Introduction}

Acute exacerbations of chronic obstructive pulmonary disease and community acquired pneumonia are two of the most frequent causes of admission to hospital in the United Kingdom. ${ }^{1}$ Antibiotics, including clarithromycin, are commonly prescribed during acute exacerbations of chronic obstructive pulmonary disease, especially in the presence of increased breathlessness, sputum volume, and purulence. ${ }^{2}$ However, their widespread use 
remains controversial as the same bacteria are often found both during exacerbations and while patients are in a stable clinical state, and proving a causative role is difficult. ${ }^{3}$ Furthermore, triggers other than bacterial infection, such as viruses and environmental factors, may be responsible for an important proportion of acute exacerbations.

Use of macrolide antibiotics in community acquired pneumonia has been consistently associated with improved short term mortality in observational studies, ${ }^{5}$ and national and international guidelines therefore recommend their use in combination with $\beta$ lactams for patients admitted to hospital. ${ }^{78}$ The improvement in short term mortality has been attributed to empirical coverage of atypical bacterial pathogens, but data suggesting improved outcomes with combinations of $\beta$ lactams and macrolides for pneumococcal pneumonia have also been reported. ${ }^{9}$ Consequently, macrolides are recommended for all patients with moderate to severe community acquired pneumonia and for patients with mild pneumonia intolerant of $\beta$ lactams. ${ }^{7}$

Widespread use of macrolide antibiotics has been accompanied by concerns about their possible deleterious effects on cardiovascular morbidity and mortality. Although initial pilot studies suggested that short term use of macrolides may have advantageous effects on cardiovascular outcomes, ${ }^{10}{ }^{11} \mathrm{a}$ subsequent retrospective study of erythromycin use in 1249 943 patients identified a significant increase in deaths from cardiovascular disease. ${ }^{12}$ Another macrolide, azithromycin, was shown to have a similar association with increased cardiovascular deaths during the time of administration. ${ }^{13}$ CLARICOR (Effect of Clarithromycin on Mortality and Morbidity in Patients with Ischemic Heart Disease trial) was a double blind, placebo controlled trial showing that a two week course of clarithromycin significantly increased cardiovascular and all cause mortality in patients with coronary heart disease but no evidence of respiratory infection. ${ }^{14}$ This finding was remarkable because the increased mortality rate persisted for three years after discontinuation of the drug. These findings were consolidated in a recent meta-analysis of 17 trials of antibiotics in coronary heart disease that showed increased long term mortality after macrolides, primarily due to increased deaths from cardiovascular disease. ${ }^{15}$

Clarithromycin is the most frequently used macrolide in the UK. ${ }^{16}$ No studies have examined the long term effect of clarithromycin on cardiovascular events and mortality in patients after acute exacerbations of chronic obstructive pulmonary disease or community acquired pneumonia.

We analysed data from two large prospective cohort studies in patients to hospital with acute exacerbations of chronic obstructive pulmonary disease and community acquired pneumonia. We hypothesised that use of clarithromycin would be associated with excess cardiovascular events and mortality beyond the time of prescription.

\section{Methods}

\section{Study populations}

This paper describes a secondary analysis of two large prospectively collected datasets. These were a multicentre observational study of patients admitted to hospital with exacerbations of chronic obstructive pulmonary disease and the Edinburgh pneumonia study cohort.

\section{Exacerbation of chronic obstructive pulmonary disease study}

The EXODUS (Exacerbations of Obstructive Lung Disease managed in UK Secondary care) dataset consists of patients over the age of 40 years admitted to one of 12 hospitals around the UK with acute exacerbations of chronic obstructive pulmonary disease between 2009 and 2011. Patients with a previous diagnosis of chronic obstructive pulmonary disease confirmed by spirometry were included if they were admitted to hospital with a primary diagnosis of an acute exacerbation. Patients with other respiratory conditions (active thoracic malignancy, asthma, and interstitial lung disease), those already on long term macrolide treatment, and those treated with primary palliative intent were excluded. Baseline patient demographics, severity of chronic obstructive pulmonary disease defined by spirometry, Medical Research Council dyspnoea score, and body mass index were recorded, as well as features and severity of exacerbation, treatment including antibiotic choice, and outcome of exacerbation.

We used national death certification data to identify all deaths and those for which a cardiovascular cause of death was recorded. We identified cardiovascular related hospital admissions when an ICD-10 (international classification of diseases, 10th revision) code denoting cardiovascular disease was used to record the cause of admission. The ICD-10 codes were I20-23 (acute myocardial infarction and acute coronary syndromes including unstable angina), I50 (cardiac failure incorporating congestive cardiac failure (I.50.0) and left ventricular failure (I50.1)), I46 (cardiac arrest), and cardiac arrhythmia (I44 (atrioventricular disorders), I45 (other conduction disorders), I47 (paroxysmal tachycardia), I48 (atrial fibrillation and flutter), I49 (other cardiac arrhythmias)). These codes do not include sinus bradycardia or sinus tachycardia as an arrhythmia.

\section{Edinburgh pneumonia study cohort}

The Edinburgh pneumonia cohort has been described elsewhere ${ }^{17}$; this study enrolled consecutive unselected patients admitted with radiologically confirmed community acquired pneumonia to NHS Lothian Hospitals in Edinburgh, UK, between 2005 and 2009. Patients were included in the study if they presented with a new infiltrate on a chest radiograph and had three or more of the following symptoms or signs: cough, sputum production, breathlessness, pleuritic chest pain, haemoptysis, fever, headache, and signs consistent with pneumonia on chest auscultation. Exclusion criteria were hospital acquired pneumonia (development of symptoms $>48$ hours after admission to or discharge from an acute care facility less than two weeks before admission), active malignancy, immunosuppression (including HIV infection and use of long term oral steroids or other forms of immunosuppressant treatment), pulmonary embolism, tuberculosis, and patients already on long term macrolide treatment or in whom active treatment was not considered appropriate (palliative care).

Two independent reviewers reviewed patients' case notes to determine the presence of cardiovascular events and classified the cause of death as cardiovascular or non-cardiovascular. Disagreements were resolved by discussion. Cardiovascular events were defined as hospital admissions or emergency department attendances with one of ST elevation myocardial infarct, non-ST elevation myocardial infarction, new or worsening congestive cardiac failure, new arrhythmia, or out of hospital cardiac arrest. 


\section{Outcome}

The primary outcome was the association between clarithromycin use and the first hospital admission due to a cardiovascular event or acute coronary syndrome (acute ST elevation myocardial infarction, non-ST elevation myocardial infarction, and unstable angina) within one year. Secondary outcomes were cardiovascular mortality and all cause mortality at one year.

\section{Clarithromycin use}

We classified all patients who received at least one dose of clarithromycin during their hospital admission as macrolide users. We compared them with patients who did not receive any macrolide antibiotics during their admission.

\section{Data analysis}

We used SPSS version 21 for statistical analyses. For propensity matching, we used the propensity matching add-on for SPSS (SPSS essentials for R and R version 2.14.2).

\section{Cox proportional hazards regression}

We used Cox proportional hazards regression to calculate crude and adjusted hazard ratios for all analyses of clarithromycin use with outcomes. We present data as adjusted hazard ratios with 95\% confidence intervals and as Cox survival curves for the primary outcome. We calculated adjusted hazard ratios after multivariable correction for age, sex, history of cardiovascular disease (previous myocardial infarction, stable angina, or coronary artery bypass grafting), previous cerebrovascular disease (stroke or transient ischaemic attack), neoplastic disease, diabetes mellitus, smoking, and use of cardiac drugs (antiplatelet agents, statins, angiotensin converting enzyme inhibitors, angiotensin II receptor blockers, calcium channel antagonists, and $\beta$ blockers). Severity of illness is associated with cardiovascular events and long term mortality, so we adjusted for it in all models by using validated severity scores: BAP-65 (elevated blood urea, altered mental status, pulse $>109$, and age $>65$ years) in the chronic obstructive pulmonary disease analysis ${ }^{18}$ and the pneumonia severity index in the community acquired pneumonia analysis. ${ }^{19}$ In addition, we adjusted for baseline severity of chronic obstructive pulmonary disease by entering per cent predicted forced expiratory volume in one second, pre-morbid MRC dyspnoea score, body mass index, and use of long term oxygen therapy into the chronic obstructive pulmonary disease models. We also adjusted chronic obstructive pulmonary disease models for use of inhaled corticosteroid, long acting $\beta$ agonist, and long acting anti-muscarinic drugs. The community acquired pneumonia analysis adjusted for requirement for mechanical ventilation or vasopressor support. As both studies included patients from more than one hospital, we included hospital as an independent variable to account for possible differences in macrolide use between hospitals. We entered the presence of chronic obstructive pulmonary disease as an independent variable in the community acquired pneumonia analysis. All models used forced entry of all available covariates to minimise residual confounding.

\section{Propensity adjusted analysis}

We used multivariable logistic regression to assess the probability that a patient would receive clarithromycin. The variables included in the propensity model were all those included in the Cox model in addition to admission temperature, acidosis, white blood cell count, and pre-admission antibiotic treatment in both cohorts. Specific variables were only available for adjustment in each individual cohort. In the acute exacerbations of chronic obstructive pulmonary disease cohort, these were admission symptoms (cough, increased sputum, sputum colour, chest pain), history of exacerbations in the previous year, and requirement for bilevel ventilation. The community acquired pneumonia analysis was also adjusted for intensive care unit admission, suspected aspiration, and Eastern Co-operative Oncology Group performance status. ${ }^{20}$

We assessed multicollinearity by using bivariate linear regression between variables and using the variance inflation factor. We regarded a variance inflation factor of less than 2.5 as excluding significant interactions.

We used this multivariable model to create a propensity score for each patient, representing the probability that an individual patient would be treated with clarithromycin. We then matched each patient treated with clarithromycin to a patient treated with other antibiotics with a similar propensity score by using greedy matching. This created two cohorts that were well matched for measured confounders (see supplementary data for the characteristics of the matched cohorts). As a sensitivity analysis to exclude strong differential effects among patients who could not be matched, we also repeated the analysis including the propensity score as a covariate in the Cox proportional hazards regression.

\section{Missing data}

We used multiple imputation to account for missing values in each database. We performed and report the multiple imputation analysis according to the recommendations of Sterne et al. ${ }^{21} \mathrm{We}$ did the analysis by using the multiple imputation function in SPSS version 21. The imputation model included all variables included in the propensity analysis for both the cohorts (these models also included the variables included in the Cox regression models). No data were missing for clarithromycin prescriptions or for the outcomes. We present analyses for five imputed models, as recommended by Sterne et al, ${ }^{21}$ with a sensitivity analysis showing an analysis of data from cases with complete data only.

\section{Sensitivity analysis}

To assess the influence of duration of antibiotic treatment, we analysed the association of clarithromycin use with cardiovascular outcomes according to the length of treatment ( $<3$ days, 3-6 days, 7 days, and $>7$ days). We did further analyses including only events occurring after 14 days and while patients were on and off treatment, to minimise immortal time bias. ${ }^{22}$ In addition, we did subgroup analyses stratifying patients according to their baseline cardiovascular risk. We used the QRISK2 score to estimate cardiovascular risk in patients without established cardiovascular disease. We calculated this as previously described, incorporating age, sex, smoking status, diabetes, family history, medical treatment, deprivation index, body mass index, systolic blood pressure, cholesterol, ethnicity, and comorbidities. ${ }^{23}$ We regarded a 10 year cardiovascular risk above $20 \%$ as high risk. To assess the influence of patients' age, we analysed the association of clarithromycin use on cardiovascular outcomes according thirds of age in the two cohorts. We tested for interaction of these potential effect modifiers as described by Altman et al. ${ }^{24}$

We also did sub-analyses in the groups receiving statins and other cardiovascular drugs on the basis of the CLARICOR data suggesting that cardiovascular drugs, particularly statins, ${ }^{25}$ could prevent the excess cardiovascular events associated with clarithromycin. We analysed the association of $\beta$ lactams and 
doxycycline with cardiovascular events in the chronic obstructive pulmonary disease cohort to ensure that any signal seen was specific to clarithromycin. We calculated the number needed to harm from the hazard ratio, as described by Altman et al. ${ }^{26}$ For all tests, we considered a two sided $\mathrm{P}$ value of less than 0.05 to be statistically significant.

\section{Results}

The EXODUS dataset included 1343 patients, and the community acquired pneumonia cohort had 1631 patients; table $1 \Downarrow$ shows their baseline characteristics. Severity measured by BAP-65 differed significantly between clarithromycin users and non-users in the chronic obstructive pulmonary disease cohort, but other severity markers and demographics were similar. Significant baseline disparities existed between clarithromycin users and non-users in the community acquired pneumonia cohort.

\section{Clarithromycin use and cardiovascular events}

During one year of follow-up, 268 patients from the chronic obstructive pulmonary disease cohort and 171 from the community acquired pneumonia cohort were admitted to hospital as a result of a cardiovascular event. Table $2 \Downarrow$ shows the nature of these events in patients treated with clarithromycin and patients not treated with clarithromycin. Figures $1 \Downarrow$ and $2 \Downarrow$ show Cox adjusted survival curves for cardiovascular events in the two cohorts.

\section{Acute exacerbations of chronic obstructive pulmonary disease cohort}

The unadjusted hazard ratio for the association of clarithromycin with cardiovascular events in the chronic obstructive pulmonary disease cohort was 1.48 (95\% confidence interval 1.13 to 1.94 ). For acute coronary syndrome, the unadjusted hazard ratio was 1.46 ( 0.93 to 2.29 ). In the multivariable analysis, clarithromycin use was associated with increased risk of cardiovascular events (hazard ratio $1.50,1.13$ to 1.97 ) and acute coronary syndrome (1.67, 1.04 to 2.68$)$.

\section{Community acquired pneumonia cohort}

In the unadjusted analysis, clarithromycin was associated with cardiovascular events (hazard ratio $1.75,1.25$ to 2.45 ) and with acute coronary syndrome $(1.81,1.08$ to 3.02$)$ in the community acquired pneumonia cohort. After adjustment for confounders in the Cox proportional hazards model, clarithromycin use was associated with an increased risk of cardiovascular events (hazard ratio $1.68,1.18$ to 2.38 ), but the association with acute coronary syndrome was no longer statistically significant $(1.65$, 0.97 to 2.80 ).

\section{Propensity adjusted analysis}

The relation between clarithromycin use in acute exacerbations of chronic obstructive pulmonary disease and cardiovascular events persisted after propensity matching (hazard ratio 1.48 , 1.02 to 2.14). We also found significant association between clarithromycin use and cardiovascular events in the community acquired pneumonia cohort after propensity matching (hazard ratio $1.58,1.08$ to 2.30 ). Incorporating the propensity score into the Cox model gave similar results for acute exacerbations of chronic obstructive pulmonary disease $(1.50,1.13$ to 1.98$)$ and community acquired pneumonia $(1.59,1.10$ to 2.29$)$. The characteristics of the cohorts produced after propensity matched are shown in the supplementary data.

\section{Cardiovascular and all cause mortality}

Inpatient mortality during acute exacerbations of chronic obstructive pulmonary disease was $6.2 \%$, and $18.8 \%$ of patients who survived to discharge died during one year's post-discharge follow-up had died by one year. Inpatient mortality in the community acquired pneumonia cohort was $8.7 \%$, and $12.8 \%$ of those who survived to discharge died during one year's post-discharge follow-up. Cardiovascular mortality at one year was $10.2 \%$ after acute exacerbations of chronic obstructive pulmonary disease and $4.4 \%$ in the community acquired pneumonia cohort.

In the chronic obstructive pulmonary disease cohort, we found a significant association between clarithromycin use and cardiovascular mortality (unadjusted hazard ratio 1.53, 1.05 to 2.23; adjusted hazard ratio $1.52,1.02$ to 2.26 ) but no association with all cause mortality (unadjusted hazard ratio $1.15,0.89$ to 1.48; adjusted hazard ratio $1.16,0.90$ to 1.51 ).

Clarithromycin use in community acquired pneumonia was not associated with a significant difference in all cause mortality (unadjusted hazard ratio $1.22,0.92$ to 1.62 ; adjusted hazard ratio $1.13,0.85$ to 1.51 ) or cardiovascular mortality (unadjusted hazard ratio $1.71,1.02$ to 2.87 ; adjusted hazard ratio $1.58,0.93$ to 2.71$)$.

\section{Timing of cardiovascular events}

We found no significantly increased risk of cardiovascular events while patients were taking clarithromycin in the chronic obstructive pulmonary disease cohort (hazard ratio 1.73, 0.71 to 4.25), but an increased risk was present after the clarithromycin course was ended (1.41, 1.05 to 1.89$)$. In the community acquired pneumonia cohort, the hazard ratio for association between clarithromycin use and cardiovascular events was 1.84 ( 0.75 to 4.51 ) during clarithromycin use and 1.66 (1.14 to 2.43$)$ after the antibiotic was stopped.

\section{Association with duration of antibiotic use}

Longer courses of clarithromycin were associated with more cardiovascular events (table $3 \Downarrow$ ). The median duration of treatment was seven days in both cohorts. Less than three days of clarithromycin treatment was not associated with cardiovascular events in the chronic obstructive pulmonary disease cohort (hazard ratio $0.89,0.50$ to 1.57 ) or the community acquired pneumonia cohort $(0.63,0.15-2.65)$, compared with patients who did not receive clarithromycin. This compared with hazard ratios of 1.73 (1.04 to 2.88) and 2.02 (1.34 to 3.04) for durations in excess of seven days in the two cohorts.

\section{Effect of age and cardiovascular risk status}

\section{Acute exacerbations of chronic obstructive} pulmonary disease cohort

In the chronic obstructive pulmonary disease cohort, clarithromycin use was associated with a significantly increased risk of cardiovascular events in patients with a previous history of cardiovascular disease (hazard ratio $1.69,1.07$ to 2.67). We stratified patients without a history of cardiovascular disease by their QRISK2 score. The hazard ratios of the effect of clarithromycin on cardiovascular events in such patients were 1.35 (0.94 to 1.95$)$ in those with a high cardiovascular risk and 0.88 ( 0.20 to 3.96$)$ in those with a low risk. The tests of interaction for these subgroups were not statistically significant $(\mathrm{P}=0.08$ and $\mathrm{P}=0.4$ comparing patients with a previously history of ischaemic heart disease with high risk and low risk patients). 
The lowest hazard ratios for cardiovascular events were in patients aged 60 or below (1.01, 0.36 to 2.91$)$. The hazard ratio was 1.47 (1.01 to 2.14) for patients aged 60-79, and a higher risk was associated with clarithromycin use in patients aged over 80 (hazard ratio $1.68,1.05$ to 2.69 ). The interaction test for age was not statistically significant $(\mathrm{P}=0.4$ and $\mathrm{P}=0.7$ comparing the oldest cohort with two younger cohorts).

\section{Community acquired pneumonia cohort}

In the community acquired pneumonia cohort, clarithromycin was most strongly associated with cardiovascular events in patients with a history of cardiovascular events (hazard ratio $1.98,1.15$ to 3.39$)$ and in those at high risk of cardiovascular disease (1.84, 1.01 to 3.36), with no increased events evident in patients classified as being at low risk of ischaemic heart disease (hazard ratio 1.08, 0.52 to 2.23) Again, the tests for interaction were not statistically significant $(\mathrm{P}>0.05$ for all comparisons). We also analysed the association of clarithromycin with cardiovascular events by age group, with the lowest hazard ratio seen in patients aged 50 or below $(0.88$, 0.28 to 2.78$)$. The hazard ratio was $1.57(0.80-3.11)$ for patients aged 50-69, and a higher risk was associated with clarithromycin use in patients aged 70 or over (hazard ratio $1.81,1.19$ to 2.76 ). The test of interaction was not statistically significant $(\mathrm{P}>0.05$ for all analyses).

\section{Use of other antibiotics}

Use of $\beta$ lactam or doxycycline was not associated with increased cardiovascular events (hazard ratios 1.06 (0.83 to 1.37 ) and 0.96 ( 0.61 to 1.51$)$, respectively) in the chronic obstructive pulmonary disease cohort compared with patients not receiving antibiotics. All patients received antibiotics in the community acquired pneumonia cohort, so we could not repeat this analysis in that cohort.

\section{Use of cardiovascular drugs}

We did an exploratory analysis of the risk of cardiovascular events in clarithromycin users who were also prescribed cardiovascular drugs. In the chronic obstructive pulmonary disease cohort, macrolide use among statin users was associated with a hazard ratio of 1.45 ( 0.81 to 2.62 ) compared with non-macrolide users. The concomitant use of clarithromycin, angiotensin converting enzyme inhibitors, and statins and of clarithromycin, statins, angiotensin converting enzyme inhibitors, and $\beta$ blockers was associated with hazard ratios of 1.13 ( 0.50 to 2.54 ) and 0.58 (0.01 to 44.7$)$, respectively. Tests of interaction compared with the treatment effect in the whole population were not statistically significant $(\mathrm{P}>0.05$ for all analyses).

Similarly, in the community acquired pneumonia cohort, cardiovascular drugs did not significantly modify the effect of clarithromycin on cardiovascular events $(\mathrm{P}>0.05$ for all interaction tests). Patients who used clarithromycin and a statin had a hazard ratio of 1.40 ( 0.73 to 2.69 ), users of clarithromycin, angiotensin converting enzyme inhibitors, and statins had a hazard ratio of 1.37 (0.48 to 3.96), and among patients using clarithromycin, statins, angiotensin converting enzyme inhibitors, and $\beta$ blockers, the hazard ratio was $1.32(0.54$ to 3.24).

\section{Missing data}

In the chronic obstructive pulmonary disease cohort, missing data accounted for less than $1 \%$ of the data. Complete datasets with no missing data in any fields were available in $84.1 \%$ of patients. No significant differences existed in characteristics or outcomes between patients with and without complete datasets, and we assumed data to be missing at random. The most frequently missing data were previous history of exacerbations (12.1\% of the cohort missing) and body mass index (12.0\%); the least frequent missing data were for temperature (three patients, $0.2 \%$ ) and white blood cell count (two patients, $0.1 \%$ ). The imputation model used did not significantly affect the results; each model gave similar results for the association between macrolide use and cardiovascular events in the chronic obstructive pulmonary disease cohort-model 1: hazard ratio 1.50 (1.13 to 1.97); model 2: 1.47 (1.11 to 1.93); model 3: 1.49 (1.13 to 1.96$)$, model 4: 1.49 (1.13 to 1.96$)$; and model 5: 1.51 (1.14 to 1.99$)$. In the model including only patients with complete data, the hazard ratio for cardiovascular events was 1.51 ( 1.10 to 2.08 ). No data were missing for the variables included in the Cox regression model in the community acquired pneumonia cohort.

\section{Number needed to harm}

For cardiovascular events in the main analysis, the number needed to harm calculated from the hazard ratio was $8(95 \%$ confidence interval 5 to 24) patients for macrolide use in acute exacerbations of chronic obstructive pulmonary disease and 11 ( 8 to 26 ) for macrolide use in community acquired pneumonia.

\section{Discussion}

This is the first study showing that use of clarithromycin in the context of exacerbations of chronic obstructive pulmonary disease and community acquired pneumonia may be associated with excess cardiovascular events that last beyond the period of prescription. Clarithromycin is the most widely used macrolide in the UK and is strongly recommended for use in patients with severe community acquired pneumonia. Previous studies have suggested an excess cardiovascular morbidity after clarithromycin use,${ }^{14}$ but these patients were selected on the basis of pre-existing cardiovascular risk rather than having a defined infective episode requiring treatment with this drug. Other studies have shown a short term association seen only during the time of administration. ${ }^{12} 13$ The observed association with cardiovascular events is of a similar magnitude to that seen in stable non-infected patients with established coronary artery disease. ${ }^{14}$ Our data suggest that the increased risk may persist beyond the time when clarithromycin is stopped.

\section{Possible explanations for findings}

Although short term events may be associated with clarithromycin's pro-arrhythmic effects mediated through prolongation of the QT interval, ${ }^{27}$ this would not affect outcome after cessation of the drug and would support an ischaemic mechanism. Clarithromycin may activate macrophages, leading to an inflammatory cascade resulting in more vulnerable plaques that over time may lead to acute coronary syndromes or sudden cardiac death by plaque rupture. ${ }^{25}$ This may explain why clarithromycin seems to increase cardiovascular events and mortality beyond the time of prescription.

We did several hypothesis generating analyses to explore the relation between clarithromycin use and cardiovascular events in these cohorts. We found a strong association between prolonged (more than seven days) courses of clarithromycin and cardiovascular events, which strengthens the case for a true biological cause. The association between duration of antibiotic treatment and cardiovascular events could also represent residual 
confounding by severity of illness. Although prolonged courses of antibiotics are common for respiratory tract infections in clinical practice, a considerable body of evidence suggests that short courses of antibiotics are equivalent to prolonged courses in community acquired pneumonia and other respiratory tract infections. ${ }^{28}$

\section{Strengths and weaknesses of study}

The strengths of this study are that data were collected in routine care from unselected hospital admissions for community acquired pneumonia or acute exacerbations of chronic obstructive pulmonary disease throughout the UK. A similar effect was seen in a previous randomised controlled study. ${ }^{14}$ This increases the likelihood that the results will be applicable to other populations.

Despite data being collected prospectively according to predefined criteria, follow-up data are observational and therefore prone to the weaknesses of this type of study. We attempted to limit bias and confounding by adjusting for all measured confounders in each database, but bias due to unrecorded factors may remain. However, the fact that the results of the propensity score matched approach show similar hazard ratios for cardiovascular events in both cohorts is reassuring.

Previous studies have shown that cardiovascular events are common sequelae of respiratory infection, including chronic obstructive pulmonary disease exacerbations and pneumonia. ${ }^{29} 30$ A potential weakness is that patients with more severe illness were more likely to be prescribed clarithromycin and that clarithromycin may therefore be a marker for more severe infection, and hence increased cardiovascular events, rather than a direct cause of cardiovascular events. We attempted to limit this by adjusting for severity according to BAP- 65 and the pneumonia severity index and by using a propensity score. Furthermore, this was a hospital based study and the results cannot be extrapolated to prescribing in primary care.

Another potential weakness is that the two cohorts recruited patients over different ranges of time. However, we do not believe that this affects the conclusion of the study, as antibiotic guidelines did not change appreciably over this time.

\section{Implications of findings}

Evidence is accumulating for long term cardiovascular risks associated with macrolides. ${ }^{14}{ }^{15}$ The role of antibiotics in exacerbations of chronic obstructive pulmonary disease is controversial, ${ }^{34}$ and no evidence from controlled trials suggest that macrolides are superior to the alternatives in this situation. As this study is observational in nature, the findings need to be validated in other datasets before recommendations to change practice can be made.

The role of macrolides in community acquired pneumonia is more complex. A large body of evidence suggests that short term mortality is reduced in patients receiving macrolides for severe community acquired pneumonia. ${ }^{5}$ No large scale randomised controlled trials have been done, and alternative agents to cover atypical pathogens are available. Our data would support the need for a high quality randomised controlled trial of macrolides with long term follow-up.

An intriguing finding of this study was that prolonged courses of clarithromycin (more than seven days) may be associated with increased risk of cardiovascular events especially in patients with a pre-existing history of coronary heart disease. This may be of particular importance given recent data supporting long term macrolide use to prevent exacerbations of chronic obstructive pulmonary disease. ${ }^{31}$

\section{Conclusion}

The use of clarithromycin in the setting of hospital admissions for infective exacerbations of chronic obstructive pulmonary disease or community acquired pneumonia may be associated with increased cardiovascular events. Our findings require validation in independent datasets, especially from primary care settings and through randomised controlled trials of macrolides with long term follow-up.

We thank Ross Archibald, University of Dundee; Louise Peet, University of Dundee; Charly Sengheiser, Doncaster Royal Infirmary; Duneesha De Fonseka, Doncaster Royal Infirmary; Gillian B Fleming, Edinburgh Royal Infirmary; Hazel Rooney, Perth Royal Infirmary; Duncan Mills, Royal Infirmary of Edinburgh; Sarah Higgins, Doncaster Royal Infirmary; John Corcoran, John Radcliffe Hospital, Oxford; Joseph Hodgson, NHS Tayside; Martin K Smith, NHS Borders; and Mudher Al-Khairalla, Doncaster Royal Infirmary for their help with data collection.

Contributors: All authors were involved in the design of the study, analysis and interpretation of results, and preparation and revision of the manuscript. All authors had full access to all of the data in the study and can take responsibility for the integrity of the data and the accuracy of the data analysis. JDC is the guarantor.

Funding: No specific funding.

Competing interests: All authors have completed the ICMJE uniform disclosure form at www.icmje.org/coi_disclosure.pdf (available on request from the corresponding author) and declare: no support from any organisation for the submitted work; no financial relationships with any organisations that might have an interest in the submitted work in the previous three years; no other relationships or activities that could appear to have influenced the submitted work.

Ethical approval: Routine collection of clinical data was approved by local Caldicott guardians and the local research ethics committee, with the requirement for informed consent waived (reference number NR/0906AB1). Additional data collection was approved by the South East Scotland research ethics committee (reference numbers S1104/15 and S1103/27).

Data sharing: No additional data available.

1 National Respiratory Training Centre. Impact of respiratory conditions: a guide for primary care organisations. NRTC, 2002

2 National Institute for Health and Clinical Excellence. Chronic obstructive pulmonary disease: management of chronic obstructive pulmonary disease in adults in primary and secondary care. NICE, 2010. (Clinical guideline 101.)

3 Monso E, Ruiz J, Rosell A, Manterola J, Fiz J, Morera J, et al. Bacterial infection in chronic obstructive pulmonary disease: a study of stable and exacerbated outpatients using the protected specimen brush. Am J Respir Crit Care Med 1995;152:1316-20.

4 White AJ, Gompertz S, Stockley RA. Chronic obstructive pulmonary disease. 6. The aetiology of exacerbations of chronic obstructive pulmonary disease. Thorax 2003;58:73-80.

5 Restrepo MI, Mortensen EM, Waterer GW, Wunderink RG, Coalson JJ, Anzueto A. Impact of macrolide therapy on mortality for patients with severe sepsis due to pneumonia. Eur Respir J 2009;1:153-9.

6 Houck PM, MacLehose RF, Niederman MS, Lowery JK. Empiric antibiotic therapy and mortality among medicare pneumonia inpatients in 10 western states: 1993, 1995, and 1997. Chest 2001;119:1420-6

7 Lim WS, Baudouin SV, George RC, Hill AT, Jamieson C, Le Jeune I, et al. BTS guidelines for the management of community acquired pneumonia in adults: update 2009. Thorax 2009;64:Siii1-55.

8 Mandell LA, Wunderink RG, Anzueto A, Bartlett JG, Campbell GD, Dean NC, et al. Infectious Diseases Society of America/American Thoracic Society consensus guidelines on the management of community-acquired pneumonia in adults. Clin Infect Dis 2007;44:S27-72.

9 Rodriguez A, Mendia A, Sirvent JM, Barcenilla F, de la Torre-Prados MV, Solé-Violán J, et al. Combination antibiotic therapy improves survival in patients with community-acquired pneumonia and shock. Crit Care Med 2007;35:1493-8.

10 Gupta S, Leatham EW, Carrington D, Mendall MA, Kaski JC, Camm AJ. Elevated C. pneumoniae antibodies, cardiovascular events, and azithromycin in male survivors of MI. Circulation 1997;96:404-7.

11 Gurfinkel E, Bozovich G, Daroca A, Beck E, Mautner B. Randomised trial of roxithromycin in non-Q-wave coronary syndromes: ROXIS pilot study. Lancet 1997;350:404-7.

12 Ray WA, Murray KT, Meredith S, Narasimhulu SS, Hall K, Stein CM. Oral erythromycin and the risk of sudden death from cardiac causes. N Engl J Med 2004;351:1089-96. 


\section{What is already known on this topic}

Clarithromycin is often used in the treatment of acute exacerbations of chronic obstructive pulmonary disease and community acquired pneumonia

Previous observational studies have suggested that cardiovascular events may be increased during treatment with clarithromycin

One randomised controlled trial showed that a short course of clarithromycin increased cardiovascular deaths in participants with coronary heart disease

The long term effects of clarithromycin on cardiovascular events when used for respiratory infection are unclear

\section{What this study adds}

The use of clarithromycin in exacerbations of chronic obstructive pulmonary disease or community acquired pneumonia may be associated with increased cardiovascular events over the following year

13 Wayne RA, Murray KT, Hall K, Arbogast PG, Stein CM. Azithromycin and the risk of cardiovascular death. N Engl J Med 2012;366:1881-90.

14 Jespersen CM, Als-Nielsen B, Damgaard M, Hansen JF, Hansen S, Helø OH, et al. Randomised placebo controlled multicentre trial to assess short term clarithromycin for patients with stable coronary heart disease: CLARICOR trial. BMJ 2006;332:22-7.

15 Gluud C, Als-Nielsen B, Damgaard M, Fischer Hansen J, Hansen S, Helø OH, et at. Clarithromycin for 2 weeks for stable coronary heart disease: 6-year follow-up of the CLARICOR randomized trial and updated meta-analysis of antibiotics for coronary heart disease. Cardiology 2008;111:280-7.

16 Chalmers JD, Singanayagam A, Akram AR, Choudhury G, Mandal P, Hill AT. Safety and efficacy of CURB65-guided antibiotic therapy in community-acquired pneumonia. $J$ Antimicrob Chemother 2011;66:416-23.

17 Chalmers JD, Singanayagam A, Murray MP, Hill AT. Prior statin use is associated with improved outcomes in community-acquired pneumonia. Am J Med 2008;121:1002-7.

18 Shorr AF, Sun X, Johannes RS, Yaitanes A, Tabak YP. Validation of a novel risk score for severity of illness in acute exacerbations of chronic obstructive pulmonary disease. Chest 2011;140:1177-83.

19 Chalmers JD, Singanayagam A, Akram AR, Mandal P, Short PM, Choudhury G, et al. Severity assessment tools for predicting mortality in hospitalised patients with community-acquired pneumonia: systematic review and meta-analysis. Thorax 2010;65:878-83.

20 Oken MM, Creech RH, Tormey DC, Horton J, Davis TE, McFadden ET, et al. Toxicity and response criteria of the Eastern Cooperative Oncology Group. Am J Clin Oncol 1982;5:649-55.

21 Sterne JA, White IR, Carlin JB, Spratt M, Royston P, Kenward MG, et al. Multiple imputation for missing data in epidemiological and clinical research: potential and pitfalls. $B M J$ 2009;338:b2393.

22 Lévesque L, Hanley JA, Kezouh A, Suissa S. Problem of immortal time bias in cohort studies: example using statins for preventing progression of diabetes. BMJ 2010;340:b508.

23 Hippisley-Cox J, Coupland C, Vinogradova Y, Robson J, Minhas R, Sheikh A, et al. Predicting cardiovascular risk in England and Wales: prospective derivation and validation of QRISK2. BMJ 2008;336:1475-82.
24 Altman DG, Bland JM. Interaction revisited: the difference between two estimates. BMJ 2003;326:219

25 Winkel P, Hilden J, Fischer Hansen J, Hildebrandt P, Kastrup J, Kolmos HJ, et al. Excess sudden cardiac deaths after short-term clarithromycin administration in the CLARICOR trial: why is this so, and why are statins protective? Cardiology 2011;118:63-7.

26 Altman DG, Andersen PK. Calculating the number needed to treat for trials where the outcome is time to an event. BMJ 1999:319:1492.

27 Bril F, Gonzalez CD, Di Girolamo G. Antimicrobial agents-associated with QT interval prolongation. Curr Drug Saf 2010;5:85-92.

28 El Moussaoui R, de Borgie CA, van den Broek P, Hustinx WN, Bresser P, van den Berk $G E$, et al. Effectiveness of discontinuing antibiotic treatment after three days versus eight days in mild to moderate-severe community acquired pneumonia: randomised, double blind study. BMJ 2006;332:1355-8.

29 Donaldson GC, Hurst JR, Smith CJ, Hubbard RB, Wedzicha JA. Increased risk of myocardial infarction and stroke following exacerbation of COPD. Chest 2010:137:1091-7.

30 Singanayagam A, Singanayagam A, Elder DH, Chalmers JD. Is community-acquired pneumonia an independent risk factor for cardiovascular disease? Eur Respir $\mathrm{J}$ 2012;39:187-96.

31 Albert RK, Connett J, Bailey WC, Casaburi R, Cooper JA Jr, Criner GJ, et al. Azithromycin for prevention of exacerbations of COPD. N Engl J Med 2011;365:689-98.

\section{Accepted: 08 February 2013}

\section{Cite this as: BMJ 2013:346:f1235}

This is an open-access article distributed under the terms of the Creative Commons Attribution Non-commercial License, which permits use, distribution, and reproduction in any medium, provided the original work is properly cited, the use is non commercial and is otherwise in compliance with the license. See: http://creativecommons.org/licenses/by$\mathrm{nc} / 2.0 /$ and http://creativecommons.org/licenses/by-nc/2.0/legalcode. 


\section{Tables}

Table 1| Baseline characteristics. Values are numbers (percentages) unless stated otherwise

\begin{tabular}{|c|c|c|c|}
\hline Characteristics & Study cohort & Clarithromycin user & Non-clarithromycin user \\
\hline COPD cohort & $(n=1343)$ & $(\mathrm{n}=\mathbf{2 8 1})$ & $(n=1062)$ \\
\hline Median (IQR) age (years) & $72(63-79)$ & $70(63-79)$ & $72(63-80)$ \\
\hline Male sex & $655(49)$ & $134(48)$ & $521(49)$ \\
\hline Active smokers & $448(33)$ & $90(32)$ & $358(34)$ \\
\hline Previous myocardial infarction & $377(28)$ & $72(26)$ & $305(29)$ \\
\hline Previous stroke & $195(15)$ & $40(14)$ & $155(15)$ \\
\hline Renal failure & $115(9)$ & $30(11)$ & $85(8)$ \\
\hline Diabetes & $203(15)$ & $34(12)$ & $169(16)$ \\
\hline Previous neoplastic disease & $171(13)$ & $40(14)$ & $131(12)$ \\
\hline Prescribed long term oxygen therapy & $122(9)$ & $21(7)$ & $101(10)$ \\
\hline Median (IQR) FEV ${ }_{1}(\%$ predicted) & $46(34-67)$ & $45(35-62)$ & $47(35-67)$ \\
\hline Median (IQR) MRC dyspnoea score & $4(3-5)$ & $4(3-5)$ & $4(3-5)$ \\
\hline Severe exacerbation: BAP65 score $>2$ & $265(20)$ & $47(17)$ & $218(21)$ \\
\hline CAP cohort & $(n=1631)$ & $(n=980)$ & $(n=651)$ \\
\hline Median (IQR) age (years) & $66(53-77)$ & $65(53-76)$ & $68(53-78)$ \\
\hline Male sex & $806(49)$ & $496(51)$ & $310(48)$ \\
\hline Active smokers & $573(35)$ & $369(38)$ & $204(31)$ \\
\hline Previous cardiovascular disease & $406(25)$ & $235(24)$ & $171(26)$ \\
\hline Previous stroke & $166(10)$ & $94(10)$ & $72(11)$ \\
\hline Liver disease & $83(5)$ & $53(5)$ & $30(5)$ \\
\hline Renal failure & $72(4)$ & $48(5)$ & $24(4)$ \\
\hline Diabetes & $152(9)$ & $107(11)$ & $45(7)$ \\
\hline Severe CAP: PSI >2 & $727(45)$ & $489(50)$ & $238(37)$ \\
\hline
\end{tabular}

BAP65=elevated blood urea, altered mental status, pulse $>109$, and age $>65$ years; $C A P=$ community acquired pneumonia; $C O P D=$ chronic obstructive pulmonary disease; $\mathrm{FEV}_{1}$ =forced expiratory volume in one second; IQR=interquartile range; $\mathrm{MRC}=$ Medical Research Council; $\mathrm{PSI}=$ =pneumonia severity index. 
Table 2| Frequency of cardiovascular events in patient cohorts. Values are numbers (percentages)

\begin{tabular}{|c|c|c|c|c|}
\hline \multirow[b]{2}{*}{ Events } & \multicolumn{2}{|c|}{ COPD cohort } & \multicolumn{2}{|c|}{ CAP cohort } \\
\hline & $\begin{array}{l}\text { Clarithromycin user } \\
\qquad(\mathrm{n}=281)\end{array}$ & $\begin{array}{l}\text { Non-clarithromycin user } \\
\qquad(n=1062)\end{array}$ & $\begin{array}{l}\text { Clarithromycin user } \\
\qquad(\mathrm{n}=980)\end{array}$ & $\begin{array}{l}\text { Non-clarithromycin user } \\
\qquad(n=651)\end{array}$ \\
\hline $\begin{array}{l}\text { Patients with } \geq 1 \text { cardiovascular } \\
\text { event }\end{array}$ & $73(26.0)$ & $195(18.4)$ & $123(12.6)$ & $48(7.4)$ \\
\hline Myocardial infarction & $12(4.3)$ & $29(2.7)$ & $25(2.6)$ & $9(1.4)$ \\
\hline $\begin{array}{l}\text { NSTEMI or acute coronary } \\
\text { syndrome }\end{array}$ & $14(5.0)$ & $40(3.8)$ & $29(3.0)$ & $11(1.7)$ \\
\hline $\begin{array}{l}\text { Congestive cardiac failure or left } \\
\text { ventricular failure }\end{array}$ & $32(11.4)$ & $56(5.3)$ & $32(3.3)$ & $21(3.2)$ \\
\hline Arrhythmia & $47(16.7)$ & $108(10.2)$ & $64(6.5)$ & $23(3.5)$ \\
\hline $\begin{array}{l}\text { Cardiac arrest/sudden cardiac } \\
\text { death }\end{array}$ & $2(0.7)$ & $3(0.3)$ & $14(1.4)$ & $6(0.9)$ \\
\hline
\end{tabular}

Sum total of events may be greater than number of patients with events, as some patients had more than one cardiovascular event. $\mathrm{CAP}=$ community acquired pneumonia; $\mathrm{COPD}=$ chronic obstructive pulmonary disease; NSTEMI=non-ST elevation myocardial infarction. 
Table 3| Effect of clarithromycin duration on cardiovascular events

\begin{tabular}{lll} 
& \multicolumn{2}{c}{ Hazard ratio $(95 \%$ Cl) for cardiovascular events } \\
\cline { 2 - 3 } Duration of treatment & \multicolumn{1}{c}{ COPD cohort } & CAP cohort \\
No clarithromycin & 1.00 (reference) & 1.00 (reference) \\
\hline$<3$ days: & & \\
\hline Unadjusted & $0.37(0.05$ to 2.70$)$ & $0.64(0.16$ to 2.63$)$ \\
\hline Adjusted & $0.89(0.50$ to 1.57$)$ & $0.63(0.15$ to 2.65$)$ \\
\hline 3-6 days: & & \\
\hline Unadjusted & $0.68(0.17$ to 2.72$)$ & $1.09(0.43$ to 2.74$)$ \\
\hline Adjusted & $0.83(0.20$ to 3.36$)$ & $1.05(0.40$ to 2.76$)$ \\
\hline 7 days: & & \\
\hline Unadjusted & $1.47(1.04$ to 2.08$)$ & $1.65(1.12$ to 2.43$)$ \\
\hline Adjusted & $1.58(1.10$ to 2.26$)$ & $1.53(1.03$ to 2.30$)$ \\
\hline$>7$ days: & & \\
\hline Unadjusted & $1.64(1.12$ to 2.39$)$ & $2.09(1.43$ to 3.07$)$ \\
\hline Adjusted & $1.73(1.04$ to 2.88$)$ & $2.02(1.34$ to 3.04$)$ \\
\hline
\end{tabular}

$\mathrm{CAP}=$ community acquired pneumonia; $\mathrm{COPD}=$ chronic obstructive pulmonary disease. 


\section{Figures}

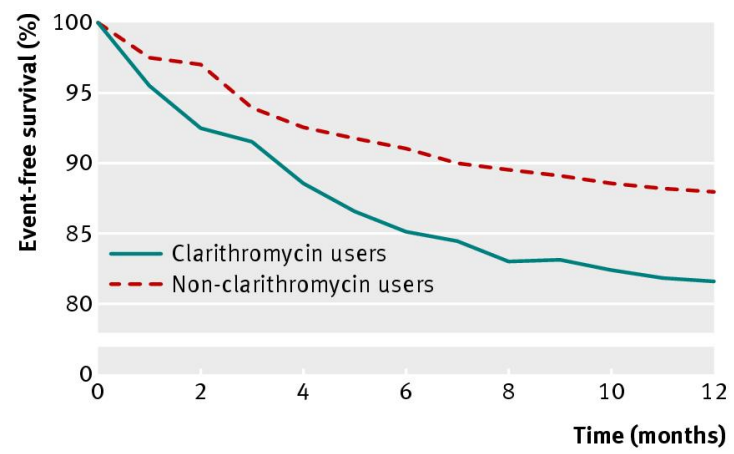

Fig 1 Cox adjusted survival curves for cardiovascular events in acute exacerbations of chronic obstructive pulmonary disease cohort

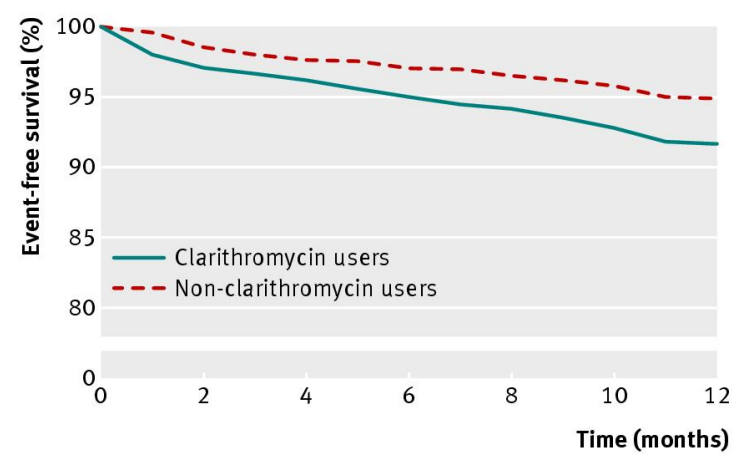

Fig 2 Cox adjusted survival curves for cardiovascular events in community acquired pneumonia cohort 